\title{
Associations between sarcopenia with asthmatic prevalence, lung function and comorbidity
}

\author{
Zhigang Hu ( $\nabla$ hxq910813@163.com ) \\ China Three Gorges University \\ Yufeng Tian \\ China Three Gorges University \\ Xinyu Song \\ Yichang Central People's Hospital \\ Fanjun Zeng \\ China Three Gorges University

\section{Ailan Yang} \\ Yichang Central People's Hospital at zhijiang
}

\section{Research Article}

Keywords: sarcopenia, asthma, prevalence, lung function, comorbidity.

Posted Date: January 13th, 2022

DOI: https://doi.org/10.21203/rs.3.rs-1223134/v1

License: (c) (1) This work is licensed under a Creative Commons Attribution 4.0 International License. Read Full License 


\section{Abstract \\ Background}

Sarcopenia was listed as a treatment trait in behavioral/risk factors of severe asthma, but studies between asthma and sarcopenia were scant. This study plans to determine the associations between sarcopenia with asthmatic prevalence, symptoms, lung function and comorbidity.

\section{Methods}

15404 individuals from the China Health and Retirement Longitudinal Study(CHARLS) and 10263 individuals from Study on global AGEing and adult health(SAGE) in China were included in this study. Four components of this study were respectively used to assess bidirectional association in the prevalence between sarcopenia with asthma, and estimate the relationships between sarcopenia with asthmatic symptoms, lung function and comorbidity via generalized additive models. The 10-item Center for Epidemiological Studies-Depression Scale $\geq 12$ scores was classified as depression in CHARLS.

\section{Results}

In CHARLS and SAGE, the prevalence of sarcopenia in asthmatics was higher than those without asthma. Asthmatics with sarcopenia had a significantly increased prevalence of severe shortness of breath(sarcopenia yes vs no, adjusted OR=3.71, 95\% Cl: 1.43-9.60) and airway obstruction in SAGE(sarcopenia yes vs no, adjusted $\mathrm{OR}=6.82,95 \% \mathrm{Cl}$ : $2.54-18.34$ ) and an obvious reduction of $\mathrm{PEF}$ in CHARLS and SAGE(sarcopenia yes vs no, adjusted RR=0.86, 95\%Cl: 0.82-0.91) compared to asthmatics without sacropenia. The presence of sarcopenia was positively associated with the prevalence of chronic obstructive pulmonary disease(sarcopenia yes vs no, adjusted $\mathrm{OR}=5.76,95 \% \mathrm{Cl}: 2.01-16.5$ ) and depression(sarcopenia yes vs no, adjusted OR=1.87, 95\%Cl:1.11-3.14) in asthmatics.

\section{Conclusions}

Our findings indicated that sarcopenia partakes in the development of asthma by affecting lung function and comorbidity and maybe considered a treatable trait of asthma management.

\section{Introduction}

In 2018, approximately 339 million individuals suffer from asthma with respiratory symptoms and sudden episode of airflow limitation worldwide. ${ }^{1}$ Frequent exacerbations of asthma puts a huge physiological and economic burden on patients and their family, even leads to disability and mortality. Worsening lung function and comorbidities(such as depression and chronic lung diseases) were two independent risk factors of asthmatic exacerbations [1]. In recent years, treatable traits are regarded as a 
new paradigm for asthma management, one key of which involves early identification of pulmonary, extrapulmonary, and behavioral/risk factors with relation to asthmatic exacerbations [2-5]. Recent study demonstrated that targeting treatable traits may reduce acute exacerbations, and improve the quality of life and asthma control in individuals with severe asthma [2].

Sarcopenia is defined as a age-related loss of skeletal muscle mass plus loss of muscle strength and/or reduced physical performance with the age cutoffs at either 60 or 65 years old in Asian Working Group for sarcopenia(AWGS) 2019 [6]. The diagnose of sarcopenia requires to have the measurement of appendicular skeletal muscle mass(ASM), muscle strength and physical performance. According to AWGS 2014 criteria, the prevalence of sarcopenia was from 5.5-25.7\% with male predominance. AWGS 2019 recommends that the cutoff of gait speed rose from $0.8 \mathrm{~m} / \mathrm{s}$ to $<1.0 \mathrm{~m} / \mathrm{s}$ [6], which may increase the prevalence of sarcopenia. Sarcopenia with muscle fiber atrophy may cause immune senescence [7], the deterioration of respiratory force generation [8] and pulmonary function [9], frailty [10], depression and mortality [10] in the general population. In the study of McDonald et al, sarcopenia was regarded as a treatment trait in behavioral/risk factors of severe asthma. However, studies about the associations between sarcopenia with asthmatic prevalence, lung function and comorbidity were very scant.

It can be hypothesized that muscle fiber atrophy and weakness secondary to sarcopenia potentially lead to the impairment of respiratory function, physical activity limitation and the increasing risk of developing depression or comorbidity in individuals with asthma. By using two national population-based studies in China, we evaluated the associations in the prevalence between sarcopenia with asthma. Subsequently, this study determined whether sarcopenia and its severity are associated with the reduction of lung function in elderly individuals with asthma. Finally, we investigated whether sarcopenia has significant relationships with comorbidities via generalized additive models.

\section{Methods}

\section{Study population}

The China Health and Retirement Longitudinal Study(CHARLS) started in between June 2011 and March 2012 and involved 17708 individuals aged more than 45 years, which represented a nationally population-based health, social and economic status with covering 450 urban or rural areas in 28 provinces of China. CHARLS uses a face-to-face computer-assisted personal interview(CAPI) with physical measurements, blood sample collection, and depression assessment. Follow-up was conducted every 2 years with the participation of new individuals in CHARLS. The Biomedical Ethics Review Committee of Peking University approved CHARLS. Written informed consents were collected in National School of Development of Peking University. More detailed description about CHARLS has been reported elsewhere [11].

Study on global AGEing and adult health(SAGE) is designed by the World Health Organization(WHO) and plans to assess and compare health status and socio-economic consequences of adult populations and the ageing process in six countries(China, Ghana, India, Mexico, Russian Federation and South Africa) 
worldwide. SAGE in China wave 1 was created between 2007 and 2010, involved 15050 individuals and covered the eight provinces. In 2012, the date involving 19 districts in Shanghai and 9524 individuals were included in SAGE. WHO Ethical Review Committee and local ethics research review boards approved ethical and obtained written informed consent. All information and data were found in elsewhere ${ }^{12}$ and the following link: https://apps.who.int/healthinfo/systems/survey data/index.php/ catalog/sage/about(accessed on 30 May 2021) [12].

\section{Definition of asthma, sarcopenia and depression}

The diagnosis of asthma was based on a positive answer of the following question: Have you ever been diagnosed with asthma by a doctor. In WHO SAGE, we collected the relevant data about asthmatic medications treatment and symptoms (attacks, awakening, and severe shortness of breath) in the past 12 months. Severe shortness of breath was based on a positive answer of the following question: Have you had an attack of shortness of breath that came on without obvious cause when you were not exercising or doing some physical activity?.

According to the recommend of AWGS 2019 [6], muscle strength and physical performance in the diagnosis of sarcopenia were measured by using handgrip strength ( $<28.0 \mathrm{~kg}$ for men and $<18.0 \mathrm{~kg}$ for women) and gait speed $(<1.0 \mathrm{~m} / \mathrm{s})$ in CHARLS and WHO SAGE. In addition, CHARLS also provided 5-time chair stand test to measure low physical performance $(\geq 12 \mathrm{~s})$. The following anthropometric equation for the height-adjusted muscle mass $\left(\mathrm{ASM} / \mathrm{Ht}^{2}\right)$ can be used to determine whether low ASM exists in Chinese population[6, 13]: $\mathrm{ASM} / \mathrm{Ht}^{2}=\left(0.193 *\right.$ body weight $+0.107 *$ height $-4.157^{*}$ gender $-0.037 *$ age2.631)/height ${ }^{2}$. Similar to previous studies

[14-16], the cut-off for defining low muscle mass was based on the ASM/Ht2 of the lowest 20 th\% percentile of the study population. Therefore, ASM/ $\mathrm{Ht}^{2}<6.92$ for men and $<5.14$ for female are regarded as low ASM. The age cutoffs of sarcopenina are set at 60 years old [6]. Low ASM with low muscle strength or physical performance were defined as sarcopenia, meanwhile patients with severe sarcopenia were associated with low ASM, muscle strength and physical performance [6].

CHARLS assessed whether depression exists by using the 10-item Center for Epidemiological StudiesDepression Scale (CES-D10). The 10 items with 4 four answers were used to estimate the depressive feelings and behaviors of individuals over one week, and value of 0-3 is assigned to each answer. Previous studies identified that CES-D10 harbors adequate reliability and validity in the assessment of depression for the community-dwelling older Chinese population [17]. CES-D10 $\geq 12$ scores with total scores of 0 to 30 scores was considered depression $[17,18]$.

\section{Variables}

In CHARLS, this study included the following demographic characteristics as adjusted confounding factors: sex, age, region, urban/rural, married status, alcohol, smoking, body mass index(BMI), night sleep duration and thirteen physician- diagnosed comorbidities (hypertension, dyslipidemia, hyperglycemia, 
cancers, chronic lung diseases, liver diseases, heart diseases, stroke, kidney diseases, digestive diseases, emotional or psychiatric problems, memory-related diseases, and arthritis or rheumatism). Lung function was measured through peak expiratory flow(PEF) in CHARLS.

In WHO SAGE, the following variables were used to adjusted the associations between asthma and sarcopenia: sex, age, region, urban/rural, married status, alcohol, smoking, vigorous-intensity activity, moderate-intensity activity, BMI, night sleep duration, hypertension, diabetes, angina, stroke, chronic lung diseases and arthritis. This study evaluated lung function in individuals with asthma through the following variables: forced expiratory volume in the first second (FEV1), FEV1/forced vital capacity(FVC), $P E F$, forced expiratory flow rate, mid-exhalation (FEF25-75\%). FEV1/FVC $<0.7$ was regarded as airway obstruction. Individuals with chronic lung diseases and $\mathrm{FEV} 1 / \mathrm{FVC}<0.7$ were diagnosed as having chronic obstructive pulmonary disease (COPD).

For Chinese adults, BMI was divided into four groups: underweight $\left(<18.5 \mathrm{~kg} / \mathrm{m}^{2}\right)$, normal $(18.5$ to $<24.0$ $\left.\mathrm{kg} / \mathrm{m}^{2}\right)$, overweight $\left(24.0\right.$ to $\left.<28.0 \mathrm{~kg} / \mathrm{m}^{2}\right)$, and obesity $\left(\geq 28.0 \mathrm{~kg} / \mathrm{m}^{2}\right)$ [19]. Age was divide into three groups: $60-69,70-79$, and 80 years. More detailed groups of all variables were shown in Table 1 and Table 2. 
Table 1

The characteristics of study population in the China Health and Retirement Longitudinal Study

\begin{tabular}{|c|c|c|c|c|}
\hline & No sarcopenia & Sarcopenia & Severe sarcopenia & $P$ \\
\hline $\mathrm{N}$ & 12159 & 2350 & 895 & \\
\hline Age & $66.7 \pm 5.6$ & $69.9 \pm 6.7$ & $73.9 \pm 7.2$ & $<0.01$ \\
\hline Year & & & & $<0.01$ \\
\hline 2011 & 3604 (29.6\%) & $877(37.3 \%)$ & $310(34.6 \%)$ & \\
\hline 2013 & $3846(31.6 \%)$ & $715(30.4 \%)$ & $259(28.9 \%)$ & \\
\hline 2015 & 4709 (38.7\%) & $758(32.3 \%)$ & $326(36.4 \%)$ & \\
\hline Sex & & & & 0.09 \\
\hline Male & $6303(51.8 \%)$ & $1199(51.0 \%)$ & $495(55.3 \%)$ & \\
\hline Female & $5856(48.2 \%)$ & $1151(49.0 \%)$ & $400(44.7 \%)$ & \\
\hline Region & & & & $<0.01$ \\
\hline Southwest & $3271(26.9 \%)$ & $924(39.3 \%)$ & $322(36.0 \%)$ & \\
\hline South and central & $6344(52.2 \%)$ & $1163(49.5 \%)$ & $447(49.9 \%)$ & \\
\hline North & $2544(20.9 \%)$ & $263(11.2 \%)$ & $126(14.1 \%)$ & \\
\hline Urban/Rural & & & & $<0.01$ \\
\hline Urban & $7232(59.5 \%)$ & $1746(74.3 \%)$ & $671(75.0 \%)$ & \\
\hline Rural & $4927(40.5 \%)$ & $604(25.7 \%)$ & $224(25.0 \%)$ & \\
\hline Married status & & & & $<0.01$ \\
\hline Current unmarried & $2021(16.6 \%)$ & $551(23.4 \%)$ & $302(33.7 \%)$ & \\
\hline Current married & $10138(83.4 \%)$ & 1799 (76.6\%) & $593(66.3 \%)$ & \\
\hline Alcohol & & & & $<0.01$ \\
\hline More than once a month & $3170(26.1 \%)$ & $605(25.7 \%)$ & $220(24.6 \%)$ & \\
\hline Less than once a month & $927(7.6 \%)$ & $144(6.1 \%)$ & $48(5.4 \%)$ & \\
\hline Never & 8062 (66.3\%) & 1601 (68.1\%) & 627 (70.1\%) & \\
\hline Smoking & & & & $<0.01$ \\
\hline Never & $6806(56.0 \%)$ & $1175(50.0 \%)$ & 456 (50.9\%) & \\
\hline Ever & $1556(12.8 \%)$ & 239 (10.2\%) & 119 (13.3\%) & \\
\hline
\end{tabular}




\begin{tabular}{|c|c|c|c|c|}
\hline & No sarcopenia & Sarcopenia & Severe sarcopenia & $P$ \\
\hline Current & 3797 (31.2\%) & $936(39.8 \%)$ & $320(35.8 \%)$ & \\
\hline Body mass index category & & & & $<0.01$ \\
\hline Underweight & $76(0.6 \%)$ & $834(35.5 \%)$ & $344(38.4 \%)$ & \\
\hline Normal & $5996(49.3 \%)$ & $1512(64.3 \%)$ & $549(61.3 \%)$ & \\
\hline Overweight & $4460(36.7 \%)$ & $4(0.2 \%)$ & $2(0.2 \%)$ & \\
\hline Obesity & $1627(13.4 \%)$ & $0(0.0 \%)$ & $0(0.0 \%)$ & \\
\hline Night sleep duration & & & & $<0.01$ \\
\hline$<360$ mins & $4000(32.9 \%)$ & $931(39.6 \%)$ & $370(41.3 \%)$ & \\
\hline $360-419$ mins & $2641(21.7 \%)$ & 459 (19.5\%) & $148(16.5 \%)$ & \\
\hline 420-479 mins & $2150(17.7 \%)$ & $308(13.1 \%)$ & $107(12.0 \%)$ & \\
\hline 480-539 mins & $2352(19.3 \%)$ & $427(18.2 \%)$ & $160(17.9 \%)$ & \\
\hline$\geq 540$ mins & $1016(8.4 \%)$ & $225(9.6 \%)$ & $110(12.3 \%)$ & \\
\hline Hypertension & & & & $<0.01$ \\
\hline No & $8392(69.0 \%)$ & $1854(78.9 \%)$ & $675(75.4 \%)$ & \\
\hline Yes & 3767 (31.0\%) & $496(21.1 \%)$ & $220(24.6 \%)$ & \\
\hline Dyslipidemia & & & & $<0.01$ \\
\hline No & $10637(87.5 \%)$ & $2185(93.0 \%)$ & $828(92.5 \%)$ & \\
\hline Yes & $1522(12.5 \%)$ & $165(7.0 \%)$ & $67(7.5 \%)$ & \\
\hline Hyperglycemia & & & & $<0.01$ \\
\hline No & $11167(91.8 \%)$ & $2243(95.4 \%)$ & $858(95.9 \%)$ & \\
\hline Yes & $992(8.2 \%)$ & $107(4.6 \%)$ & $37(4.1 \%)$ & \\
\hline Cancer & & & & 0.69 \\
\hline No & $12054(99.1 \%)$ & $2331(99.2 \%)$ & $885(98.9 \%)$ & \\
\hline Yes & $105(0.9 \%)$ & $19(0.8 \%)$ & $10(1.1 \%)$ & \\
\hline Chronic lung diseases & & & & $<0.01$ \\
\hline No & $10846(89.2 \%)$ & 1999 (85.1\%) & $773(86.4 \%)$ & \\
\hline Yes & $1313(10.8 \%)$ & 351 (14.9\%) & $122(13.6 \%)$ & \\
\hline Liver diseases & & & & 0.002 \\
\hline
\end{tabular}




\begin{tabular}{|c|c|c|c|c|}
\hline & No sarcopenia & Sarcopenia & Severe sarcopenia & $P$ \\
\hline No & $11608(95.5 \%)$ & $2271(96.6 \%)$ & $871(97.3 \%)$ & \\
\hline Yes & $551(4.5 \%)$ & $79(3.4 \%)$ & $24(2.7 \%)$ & \\
\hline Heart diseases & & & & $<0.01$ \\
\hline No & $10316(84.8 \%)$ & $2069(88.0 \%)$ & $786(87.8 \%)$ & \\
\hline Yes & $1843(15.2 \%)$ & $281(12.0 \%)$ & $109(12.2 \%)$ & \\
\hline Stroke & & & & 0.02 \\
\hline No & $11821(97.2 \%)$ & 2308 (98.2\%) & $867(96.9 \%)$ & \\
\hline Yes & $338(2.8 \%)$ & $42(1.8 \%)$ & $28(3.1 \%)$ & \\
\hline Kidney diseases & & & & 0.48 \\
\hline No & $11329(93.2 \%)$ & $2199(93.6 \%)$ & $842(94.1 \%)$ & \\
\hline Yes & $830(6.8 \%)$ & $151(6.4 \%)$ & $53(5.9 \%)$ & \\
\hline Digestive diseases & & & & 0.01 \\
\hline No & $9527(78.4 \%)$ & $1785(76.0 \%)$ & $680(76.0 \%)$ & \\
\hline Yes & $2632(21.6 \%)$ & $565(24.0 \%)$ & $215(24.0 \%)$ & \\
\hline Emotional, nervous, or psyc & iatric problems & & & 0.10 \\
\hline No & $11997(98.7 \%)$ & $2327(99.0 \%)$ & $889(99.3 \%)$ & \\
\hline Yes & $162(1.3 \%)$ & $23(1.0 \%)$ & $6(0.7 \%)$ & \\
\hline Memory-related diseases & & & & 0.76 \\
\hline No & $11907(97.9 \%)$ & $2306(98.1 \%)$ & $875(97.8 \%)$ & \\
\hline Yes & $252(2.1 \%)$ & $44(1.9 \%)$ & $20(2.2 \%)$ & \\
\hline Arthritis or rheumatism & & & & 0.34 \\
\hline No & $8044(66.2 \%)$ & $1584(67.4 \%)$ & $607(67.8 \%)$ & \\
\hline Yes & $4115(33.8 \%)$ & $766(32.6 \%)$ & $288(32.2 \%)$ & \\
\hline Asthma & & & & $<0.01$ \\
\hline No & $11671(96.0 \%)$ & $2228(94.8 \%)$ & $846(94.5 \%)$ & \\
\hline Yes & $488(4.0 \%)$ & $122(5.2 \%)$ & $49(5.5 \%)$ & \\
\hline Depression & & & & $<0.01$ \\
\hline No & 8847 (72.8\%) & $1551(66.0 \%)$ & $588(65.7 \%)$ & \\
\hline
\end{tabular}




\begin{tabular}{|lllll|}
\hline & No sarcopenia & Sarcopenia & Severe sarcopenia & $P$ \\
\hline Yes & $3312(27.2 \%)$ & $799(34.0 \%)$ & $307(34.3 \%)$ & \\
\hline PEF & $289.5 \pm 119.5$ & $237.5 \pm 108.7$ & $195.9 \pm 105.3$ & $<0.01$ \\
\hline
\end{tabular}


Table 2

The characteristics of study population in the Study on global AGEing and adult health from China

\begin{tabular}{|c|c|c|c|c|}
\hline & No sarcopenia & Sarcopenia & Severe sarcopenia & $P$ \\
\hline N & 8998 & 753 & 512 & \\
\hline Age & $68.8 \pm 6.9$ & $73.0 \pm 7.2$ & $76.1 \pm 7.3$ & $<0.01$ \\
\hline Sex & & & & $<0.01$ \\
\hline Male & 4506 (50.1\%) & $312(41.4 \%)$ & 199 (38.9\%) & \\
\hline Female & 4492 (49.9\%) & $441(58.6 \%)$ & $313(61.1 \%)$ & \\
\hline Region & & & & 0.01 \\
\hline North & 2754 (30.6\%) & $195(25.9 \%)$ & $141(27.5 \%)$ & \\
\hline South & $6244(69.4 \%)$ & $558(74.1 \%)$ & 371 (72.5\%) & \\
\hline Urban/Rural & & & & $<0.01$ \\
\hline Urban & 4985 (55.4\%) & 297 (39.4\%) & 151 (29.5\%) & \\
\hline Rural & $4013(44.6 \%)$ & $456(60.6 \%)$ & 361 (70.5\%) & \\
\hline Married status & & & & $<0.01$ \\
\hline Current unmarried & 1790 (19.9\%) & $253(33.6 \%)$ & $205(40.0 \%)$ & \\
\hline Current married & 7208 (80.1\%) & $500(66.4 \%)$ & $307(60.0 \%)$ & \\
\hline Alochol & & & & 0.05 \\
\hline Ever & 2349 (26.1\%) & $175(23.2 \%)$ & $115(22.5 \%)$ & \\
\hline Never & 6649 (73.9\%) & $578(76.8 \%)$ & 397 (77.5\%) & \\
\hline Smoking & & & & 0.73 \\
\hline Never & $6302(70.0 \%)$ & $522(69.3 \%)$ & 371 (72.5\%) & \\
\hline Ever & 888 (9.9\%) & $72(9.6 \%)$ & $44(8.6 \%)$ & \\
\hline Current & 1808 (20.1\%) & $159(21.1 \%)$ & $97(18.9 \%)$ & \\
\hline Body mass index & & & & $<0.01$ \\
\hline Underweight & $131(1.5 \%)$ & $231(30.7 \%)$ & $146(28.5 \%)$ & \\
\hline Normal & 3992 (44.4\%) & $521(69.2 \%)$ & $364(71.1 \%)$ & \\
\hline Overweight & 3525 (39.2\%) & $1(0.1 \%)$ & $2(0.4 \%)$ & \\
\hline Obesity & 1350 (15.0\%) & $0(0.0 \%)$ & $0(0.0 \%)$ & \\
\hline
\end{tabular}




\begin{tabular}{|c|c|c|c|c|}
\hline & No sarcopenia & Sarcopenia & Severe sarcopenia & $P$ \\
\hline Moderate-intensity activity & & & & $<0.01$ \\
\hline Yes & $2840(31.6 \%)$ & $304(40.4 \%)$ & $188(36.7 \%)$ & \\
\hline No & $6158(68.4 \%)$ & $449(59.6 \%)$ & $324(63.3 \%)$ & \\
\hline Vigorous-intensity activity & & & & 0.61 \\
\hline Yes & $748(8.3 \%)$ & $66(8.8 \%)$ & $37(7.2 \%)$ & \\
\hline No & $8250(91.7 \%)$ & $687(91.2 \%)$ & $475(92.8 \%)$ & \\
\hline Night sleep duration & & & & $<0.01$ \\
\hline$<360$ mins & $751(8.3 \%)$ & $79(10.5 \%)$ & $62(12.1 \%)$ & \\
\hline $360-419$ mins & $1210(13.4 \%)$ & $92(12.2 \%)$ & $53(10.4 \%)$ & \\
\hline $420-479$ mins & $1958(21.8 \%)$ & $126(16.7 \%)$ & $58(11.3 \%)$ & \\
\hline 480-539mins & $2890(32.1 \%)$ & $224(29.7 \%)$ & $122(23.8 \%)$ & \\
\hline$\geq 540 \mathrm{~min}$ & $2189(24.3 \%)$ & $232(30.8 \%)$ & $217(42.4 \%)$ & \\
\hline Hypertension & & & & $<0.01$ \\
\hline No & $5359(59.6 \%)$ & $586(77.8 \%)$ & $403(78.7 \%)$ & \\
\hline Yes & $3639(40.4 \%)$ & $167(22.2 \%)$ & $109(21.3 \%)$ & \\
\hline Diabetes & & & & $<0.01$ \\
\hline No & $8082(89.8 \%)$ & $718(95.4 \%)$ & $489(95.5 \%)$ & \\
\hline Yes & $916(10.2 \%)$ & $35(4.6 \%)$ & $23(4.5 \%)$ & \\
\hline Stroke & & & & 0.22 \\
\hline No & $8051(89.5 \%)$ & $685(91.0 \%)$ & $467(91.2 \%)$ & \\
\hline Yes & $947(10.5 \%)$ & $68(9.0 \%)$ & $45(8.8 \%)$ & \\
\hline Angina & & & & 0.32 \\
\hline No & $8486(94.3 \%)$ & $720(95.6 \%)$ & $483(94.3 \%)$ & \\
\hline Yes & $512(5.7 \%)$ & $33(4.4 \%)$ & $29(5.7 \%)$ & \\
\hline Chronic lung diseases & & & & $<0.01$ \\
\hline No & $8101(90.0 \%)$ & $654(86.9 \%)$ & $434(84.8 \%)$ & \\
\hline Yes & 897 (10.0\%) & $99(13.1 \%)$ & $78(15.2 \%)$ & \\
\hline Arthritis & & & & 0.01 \\
\hline
\end{tabular}




\begin{tabular}{|lllll|}
\hline & No sarcopenia & Sarcopenia & Severe sarcopenia & $P$ \\
\hline No & $6879(76.5 \%)$ & $615(81.7 \%)$ & $376(73.4 \%)$ & \\
\hline Yes & $2119(23.5 \%)$ & $138(18.3 \%)$ & $136(26.6 \%)$ & \\
\hline Asthma & & & & 0.1 \\
\hline No & $8733(97.1 \%)$ & $724(96.1 \%)$ & $490(95.7 \%)$ & \\
\hline Yes & $265(2.9 \%)$ & $29(3.9 \%)$ & $22(4.3 \%)$ & \\
\hline FEV1 & $1.7 \pm 0.7$ & $1.5 \pm 0.7$ & $1.3 \pm 0.7$ & $<0.01$ \\
\hline FEV1/FVC & & & & $<0.01$ \\
\hline$\geq 0.7$ & $7252(80.6 \%)$ & $555(73.7 \%)$ & $352(68.8 \%)$ & \\
\hline$<0.7$ & $1746(19.4 \%)$ & $198(26.3 \%)$ & $160(31.2 \%)$ & \\
\hline PEF & $78.6 \pm 19.9$ & $76.6 \pm 19.5$ & $73.8 \pm 21.0$ & $<0.01$ \\
\hline FEF25-75 & $2.0 \pm 1.1$ & $1.6 \pm 1.1$ & $1.3 \pm 1.0$ & $<0.01$ \\
\hline
\end{tabular}

\section{Statistical analysis}

Study populations were categorized into three groups: no sarcopenia, non-severe sarcopenia and severe sarcopenia. SPSS described categorical variables by counts and percentages (\%), subsequently compared the difference between three groups via a chi-square test. Continuous variables were presented as means and standard deviations with Mann-Whitney $U$ test for skewed continuous variables and Student's t test or one-way ANOVA for normally distributed continuous variables. Chi-square goodness-offit method tested the normality of distribution of the data. The first component of the study assessed the associations in the prevalence between sarcopenia and asthma via three generalized additive models with binomial regression. The adjusted variables of each model were shown in each table. Model 1 included patient demographic characteristics, model 2 added physical/behavioral factors, and model 3 added physical/behavioral factors and comorbidities. In CHARLS, the least absolute shrinkage and selection operator (LASSO) [20] and multivariate logistic analyses with binomial regression were used to screen the independent risk factors of sarcopenia in asthmatics. We also evaluated the relationships between sarcopenia with asthmatic medications and symptoms in the second component. Model 4 also included lung function on the basis of model 3 in Table 3 . The third component was that three generalized additive models with Possion regression were used to compare the differences of lung function among three sarcopenia groups in asthmatics. In WHO SAGE, model 4 added the adjustments of asthmatic medications and symptoms. Finally, three generalized additive models with binomial regression were used to evaluate the associations between sarcopenia with depression and COPD in asthmatics. All statistical analyses were done in SPSS, Empower(R) (www. empowerstats.com; X\&Y solutions, Inc., Boston MA). Odd ratios (ORs) for binomial regression and analysis and rate ratio(RR) for 
Poisson regression analysis with $95 \%$ confidence intervals (Cls) represented the strength of association, meanwhile a two tailed $\mathrm{P}<0.05$ was considered statistically significant.

Table 3

The associations between sarcopenia and asthma-related symptoms in the Study on global AGEing and adult health from China

\begin{tabular}{|c|c|c|c|c|}
\hline & Model 1 & Model 2 & Model 3 & Model 4 \\
\hline \multicolumn{5}{|c|}{ Asthma-related attacks } \\
\hline $\begin{array}{l}\text { Sarcopenia(no vs } \\
\text { yes) }\end{array}$ & $0.97(0.40,2.38)$ & $0.82(0.32,2.06)$ & $0.72(0.28,1.86)$ & $0.53(0.19,1.51)$ \\
\hline \multicolumn{5}{|c|}{ Asthma-related awakening } \\
\hline $\begin{array}{l}\text { Sarcopenia(no vs } \\
\text { yes) }\end{array}$ & $1.72(0.69,4.31)$ & $1.97(0.74,5.26)$ & $2.02(0.73,5.59)$ & $2.66(0.90,7.82)$ \\
\hline \multicolumn{5}{|c|}{ Asthma-related severe shortness of breath } \\
\hline $\begin{array}{l}\text { Sarcopenia(no vs } \\
\text { yes) }\end{array}$ & $\begin{array}{l}3.51(1.50 \\
8.20)^{\#}\end{array}$ & $\begin{array}{l}3.63(1.50 \\
8.79)^{\#}\end{array}$ & $\begin{array}{l}3.41(1.38 \\
8.42)^{\#}\end{array}$ & $\begin{array}{l}3.71(1.43, \\
9.60)^{\#}\end{array}$ \\
\hline \multicolumn{5}{|c|}{$\begin{array}{l}\text { Model } 1 \text { adjusted the following variables: sex, age, region, urban/rural, married status and body mass } \\
\text { index, }\end{array}$} \\
\hline \multicolumn{5}{|c|}{$\begin{array}{l}\text { Model } 2 \text { adjusted the following variables: sex, age, region, urban/rural, married status, body mass } \\
\text { index, alcohol, smoking, vigorous-intensity activity, moderate- intensity activity and night sleep } \\
\text { duration. }\end{array}$} \\
\hline \multicolumn{5}{|c|}{$\begin{array}{l}\text { Model } 3 \text { adjusted the following variables: sex, age, region, urban/rural, married status,body mass } \\
\text { index, alcohol, smoking, vigorous-intensity activity, moderate- intensity activity, night sleep duration, } \\
\text { hypertension, diabetes, angina, stroke, chronic lung diseases, and arthritis. }\end{array}$} \\
\hline \multicolumn{5}{|c|}{$\begin{array}{l}\text { Model } 4 \text { adjusted the following variables: sex, age, region, urban/rural, married status, alcohol, } \\
\text { smoking, vigorous-intensity activity, moderate-intensity activity, body mass index, night sleep duration, } \\
\text { hypertension, diabetes, angina, stroke, chronic lung diseases, arthritis, FEV1, airway obstruction, PEF, } \\
\text { and FEF } 25 \%-75 \%) \text {. }\end{array}$} \\
\hline$\# P<0.01$ & & & & \\
\hline
\end{tabular}

\section{Results}

\section{The characteristics in CHARLS and WHO SAGE}

In three cycles of CHARLS (2011, 2013 and 2015), a total of 15404 individuals aged 67.6 \pm 6.2 years (range, 60-103 years) were included in this present study. More than half $(51.9 \%)$ of individuals were male with predominantly never alcohol and smoking. The overall prevalence of sarcopenia was $21.1 \%$ with $5.8 \%$ of severe sarcopenia. The prevalence of sarcopenia showed an upward trend with the increase of age: $14.4 \%, 31.8 \%$, and $51.2 \%$ in the $60-69,70-79$ and $\geq 80$ years groups, respectively. $659(4.3 \%)$ 
individuals reported physician-diagnosed asthma. 4433 (28.7\%) individuals were classified as depressive group (CES-D $10 \geq 12$ scores).

A total of 10263 individuals with $69.5 \pm 7.2$ years(range, 60-102 years) were selected from WHO SAGE. Predominantly female (51.1\%) with high-proportioned never alcohol and smoking were shown in WHO SAGE. The overall prevalence of sarcopenia was $12.3 \%$ with $10.2 \%$ among male and $14.4 \%$ among female. 512(5\%) individuals were diagnosed with severe sarcopenia. Prevalence of sarcopenia in three age groups also significantly increased and varied by $6.0 \%, 16.4 \%$ and $32.5 \% .2104$ (20.5\%) individuals had airway obstruction(FEV1/FVC<0.7). The prevalence of asthma was 3.1\% (316 individuals). 301 asthmatics provided the information about medications treatment $(73.4 \%)$ and asthma-related symptoms. The proportions of asthma-related attacks, awakening, and activity limitation were $68.1 \%$, $27.2 \%$ and $42.2 \%$, respectively. More detailed data were shown in Table 1 and Table 2.

\section{Associations in the prevalence between sarcopenia with asthma}

In CHARLS, the prevalence of non-severe and severe sarcopenia among asthmatics were $18.5 \%$ and $7.4 \%$, respectively(see Figure 1). $4.0 \%$ of individuals with sarcopenia had the diagnosis of asthma, which was higher than those of individuals without sarcopenia(2.9\%, $P=0.036)$. Model 3 indicated that sarcopenia and asthma have no significantly bidirectional association in the prevalence(see table S1). In asthmatics, LASSO and multivariate logistic regression analyses indicated that aged with rural (rural vs unban, adjusted OR=0.41,95\% Cl: 0.23-0.75) and depression (CES-D10 < 12 scores vs $\geq 12$ scores, adjusted $\mathrm{OR}=1.95,95 \% \mathrm{Cl}: 1.16-3.27)$ harbored relatively high prevalence of sarcopenia.

In WHO SAGE, $9.2 \%$ and $7 \%$ of individuals with asthma could be diagnosed with non-severe and severe sarcopenia, which were higher than those in individuals without asthma(see Figure 1). The prevalence of asthma was $4.0 \%$ in individuals with sarcopenia. Three models observed no significantly association in the prevalence between asthma and sarcopenia(see table S2). LASSO and multivariate logistic regression analyses suggested that female, rural, aged and airway obstruction were indepedent risk factors of sarcopenia among asthmatics.

\section{Sarcopenia and asthma-related symptoms}

Four models also showed that asthma-related attacks and awakening had no significant associations with the presence of sarcopenia (see Table 3). However, sarcopenia was associated with significantly higher prevalence of asthma-related severe shortness of breath after adjusted demographic characteristics, physical/behavioral factors, comorbidities and lung function (adjusted OR=3.71, 95\% $\mathrm{Cl}$ : 1.43-9.60). The prevalence of sarcopenia demonstrated a rising tendency with the increasing numbers of asthma-related symptoms(see Figure 2).

\section{Sarcopenia and lung function in asthmatics}

In CHARLS, Possion regression analysis suggested that adjusted mean value of PEF were significantly declined in asthmatics with non-severe(adjusted $\mathrm{RR}=0.926,95 \% \mathrm{Cl}$ : 0.910-0.934) and severe 
sarcopenia(adjusted RR=0.759, 95\%Cl: 0.739-779) compared to no sarcopenia.

In WHO SAGE, four Possion regression analyses demonstrated that the more severe sarcopenia in asthmatics, the more obvious was lung function impairment (see Table 4 and Figure 3 ). Asthmatics with sarcopenia(adjusted $\mathrm{RR}=6.82,95 \% \mathrm{Cl}$ : $2.54-18.34$ in model 4) were associated with significantly higher prevalence of airway obstruction than those without sarcopenia. Sarcopenia had a significantly negative correlation with adjusted mean value of PEF in asthmatics(adjusted $\mathrm{RR}=0.86,95 \% \mathrm{Cl}$ : 0.82-0.91 in model 4).

Table 4

The associations between sarcopenia and lung function of asthmatics in the Study on global AGEing and adult health from China

\begin{tabular}{|c|c|c|c|c|}
\hline & Model 1 & Model 2 & Model 3 & Model 4 \\
\hline \multicolumn{5}{|l|}{ FEV1 } \\
\hline $\begin{array}{l}\text { Sarcopenia(no vs } \\
\text { yes) }\end{array}$ & $0.75(0.57,1.01)$ & $0.75(0.50,1.12)$ & $0.80(0.53,1.20)$ & $0.81(0.53,1.22)$ \\
\hline \multicolumn{5}{|l|}{ FEV1/FVC<0.7 } \\
\hline $\begin{array}{l}\text { Sarcopenia(no vs } \\
\text { yes) }\end{array}$ & $\begin{array}{l}5.44(2.25 \\
13.19)^{\#}\end{array}$ & $\begin{array}{l}5.89(2.34 \\
14.82)^{\#}\end{array}$ & $\begin{array}{l}5.37(2.09 \\
13.80)^{\#}\end{array}$ & $\begin{array}{l}\text { 6.82(2.54, } \\
18.34)^{\#}\end{array}$ \\
\hline \multicolumn{5}{|l|}{ PEF } \\
\hline $\begin{array}{l}\text { Sarcopenia(no vs } \\
\text { yes) }\end{array}$ & $0.87(0.82,0.91)^{\#}$ & $0.87(0.83,0.92)^{\#}$ & $0.89(0.85,0.94)^{\#}$ & $0.86(0.82,0.91)^{\#}$ \\
\hline \multicolumn{5}{|l|}{ FEF25\%-75\% } \\
\hline $\begin{array}{l}\text { Sarcopenia(no vs } \\
\text { yes) }\end{array}$ & $0.89(0.61,1.30)$ & $0.88(0.60,1.29)$ & $0.96(0.66,1.42)$ & $0.93(0.63,1.38)$ \\
\hline \multicolumn{5}{|c|}{$\begin{array}{l}\text { Model } 1 \text { adjusted the following variables: sex, age, region, urban/rural, married status and body mass } \\
\text { index, }\end{array}$} \\
\hline \multicolumn{5}{|c|}{$\begin{array}{l}\text { Model } 2 \text { adjusted the following variables: sex, age, region, urban/rural, married status, body mass } \\
\text { index, alcohol, smoking, vigorous-intensity activity, moderate- intensity activity and night sleep } \\
\text { duration. }\end{array}$} \\
\hline \multicolumn{5}{|c|}{$\begin{array}{l}\text { Model } 3 \text { adjusted the following variables: sex, age, region, urban/rural, married status, body mass } \\
\text { index, alcohol, smoking, vigorous-intensity activity, moderate- intensity activity, night sleep duration, } \\
\text { hypertension, diabetes, angina, stroke, chronic lung diseases, and arthritis. }\end{array}$} \\
\hline \multicolumn{5}{|c|}{$\begin{array}{l}\text { Model } 4 \text { adjusted the following variables: sex, age, region, urban/rural, married status, alcohol, } \\
\text { smoking, vigorous-intensity activity, moderate-intensity activity, body mass index, night sleep duration } \\
\text { hypertension, diabetes, angina, stroke, chronic lung diseases, arthritis, asthma related medications } \\
\text { and symptoms (attacks, awakenings and activity limitation). }\end{array}$} \\
\hline$\# P<0.01$ & & & & \\
\hline
\end{tabular}




\section{Sarcopenia and comorbidities in asthmatics}

In CHARLS, Adjusted OR values in the prevalence of depression respectively were 1.82(95\% Cl: 1.04-3.18) in nonsevere sarcopenia and 2.02 (95\% Cl: 1.01-4.10) in severe sarcopenia compared with no sarcopenia after adjusted demographic characteristics, physical/behavioral factors, and thirteen comorbidities. The presence of sarcopenia was associated with significantly higher prevalence of depression (adjusted OR=1.87, 95\%Cl:1.11-3.14 in model 3).

In WHO SAGE, sarcopenia was an idepedent risk factor of COPD in individuals with asthma(adjusted $\mathrm{OR}=5.76,95 \% \mathrm{Cl}: 2.01-16.5$ in model 4). When model 3 added the adjustment of asthmatic medication and symptoms, the prevalence of COPD in nonsevere sarcopenia group(adjusted OR=9.11, 95\%Cl:2.69-30.8 in model 4) was significantly higher than that in no sarcopenia group.

\section{Discussion}

The study used two national population-based survey to determine the

associations between sarcopenia with asthma and found the following results: Firstly, the prevalence of sarcopenia and severe sarcopenia defined by AWGS 2019 was $12.3 \%-21.3 \%$ and $5 \%-5.8 \%$ in Chinese older asthmatics. There was no significantly bidirectional association in the prevalence between asthma and sarcopenia, but an upward trend of sarcopenia was associated with the increasing numbers of asthmarelated symptoms. Secondly, the presence of sarcopenia, especially severe sarcopenia, was accompanied by a significantly increasing risk of airway obstruction and the obvious reduction of PEF. Thirdly, sarcopenia was positively associated with the prevalence of depression and COPD in terms of asthmatic comorbidity.

Studies about sarcopenia and asthma did not obtain enough attention and are very scant. This is the first study to estimate the prevalence of sarcopenia in Chinese population with asthma using AWGS 2019 criteria. Our studied demonstrated that approximately $17.6 \%$ and $5.5 \%$ of asthmatics may be diagnosed with sarcopenia and severe sarcopenia. The prevalence of sarcopenia in CHARLS was higher than that in WHO SAGE. A possible explanation was that more individuals came from rural in CHARLS. Previous studies have fully explored the adverse effects of sarcopenia on chronic obstruction pulmonary diseases[21, 22] and health status in the general population [23, 24]. Our study suggested that the prevalence of sarcopenia among asthmatics was higher than that among individuals without asthma. The screen and study of sarcopenia in asthmatics should not be neglected, especially for aging individuals with rural, depression and airway obstruction.

Persistent airway obstruction and lung function impairment are two important hallmarks of asthmatic deterioration [1]. Airway obstruction and declined lung function not only augment the risk of asthmatic exacerbations and reduce the quality of life, but also increase the difficulty of asthmatic medication treatment. ${ }^{1}$ Short-term and long-term PEF monitoring may know expiratory airflow limitation and 
contribute to early detection of asthmatic exacerbations and the assessment of asthma control level. Sarcopenia was found to have positive correlation with the prevalence of airway obstructive and the decline of PEF in our study. We also observed that asthmatics with sarcopenia were more prone to develop COPD than those without sarcopenia. It is reasonable to expect that sarcopenia along with systemic muscle fiber atrophy and weakness will inevitably lead to functional disability of respiratory muscles. The cross-talk between sarcopenia and adverse health consequences is mediated through complex mechanisms and pathways, including microRNAs, inflammatory processes, oxidative stress and etc [25-27]. The onset and development of asthma were known to involve the regulatory of microRNAs and inflammatory processes [28, 29]. In asthmatics, the expression levels of miR-21 and miR-34 had strongly negative associations with handgrip strength and ASM, whereas miR-133 and miR-206 showed significantly positive correlations with handgrip strength and ASM[30]. Sarcopenia along with low handgrip strength and ASM potentially reduces the levels of miR-133 and miR-206 in asthmatics. The decreasing miR-133a could up-regulate RhoA expression of bronchial smooth muscle in asthma model, which may lead to an augmentation of bronchial smooth muscle contraction and induce airway hyperresponsiveness(AHR) in individuals with asthma [31]. In 4,4'-methylene diphenyl diisocyanate induced asthma model, the reduction of miR-206-3p might increase inducible nitric oxide synthase transcription expression by targeting calcineurin/NFAT signaling, in turn leading to AHR. Increasing level of miR-21 not only promotes the differentiation of T cells towards Th2 in eosinophilic asthma, but also restrains HDAC2 levels and results in glucocorticoids insensitivity by up-regulated PI3K-mediated phosphorylation and nuclear translocation of pAKT in neutrophilic asthma [28]. Multiple studies have shown that sarcopenia is associated with the significant increase of pro-inflammatory cytokines, including TNF-a, IL-6, and C-reactive protein [32]. Taken together, microRNAs regulatory network and inflammatory processes can be considered the underlying pathophysiology linking sarcopenia with asthma.

Depression is classed as an important independent risk factor of asthma onset, exacerbations and mortality [1]. Our study suggested a bidirectional association between depression and sarcopenia in asthmatics, similar to the results in the general population[33, 34]. Sarcopenia, especially severe sarcopenia, was associated with significant increase of depression compared with no sarcopenia. Sarcopenia and depression harbor common pathophysiological pathways with regard to inflammation processes, neurotrophins and oxidative stress [33]. In addition, the onset of sarcopenia and depression may attribute to some same lifestyle behaviors, such as low physical activity, smoking, and malnutrition [33]. Depression caused by sarcopenia was likely to participate in the occurrence and development of asthma.

Our main strength is to firstly assess the relationships between sarcopenia with asthmatic prevalence, lung function and comorbidity in Chinese population by using two national population-based studies. However, several limitations also exist in our study. Firstly, the diagnoses of asthma and some comorbidities depend on the questionnaire's results with potential selection bias. Secondly, an 
anthropometric equation, instead of Dual X-ray absorpometry (DXA) or Bioelectrical impedance analysis(BIA), is used to evaluate ASM. However, this equation has previously been validated in Chinese population [13]. Our cutoff of low ASM was substantially identical to that in the study of Wu and the colleagues [16]. Besides, the unlity of anthropometric equation to assess low ASM may obtain a costeffective alternative to BIA or DXA to improve the diagnsis of sarcopenia, especially in large-sample population-based studies [35]. Thirdly, the cross-sectional nature of two studies restrains the capability to infer interactional causation between asthma and sarcopenia.

\section{Conclusion}

This study reports the prevalence of sarcopenia and severe sarcopenia in Chinese older population and asthmatics. Sarcopenia can contribute to the development of asthma by affecting lung function and comorbidity and maybe considered treatable traits of asthma management. Considering the high prevalence and significant effect of sarcopenia, It's worth making a routine screening for asthmatics.

\section{Abbreviations}

AHR - airway hyperresponsiveness

ASM - Appendicular skeletal muscle mass

AWGS - Asian Working Group for sarcopenia

BIA - Bioelectrical impedance analysis

BMI - Body mass index

CAPI - Computer-assisted personal interview

CHARLS - The China Health and Retirement Longitudinal Study

$\mathrm{Cl}$ - Confidence interval

DXA - Dual X-ray absorpometry

LASSO - least absolute shrinkage and selection operator

OR - Odds ratio

RR- Rate ratio

SAGE - Global Ageing and Adult Health

\section{Declarations}




\section{Ethics approval and consent to participate}

The study procedures were carried out by the Declaration of Helsinki. Written informed consents were collected in National School of Development of Peking University on the China Health and Retirement Longitudinal Study(CHARLS). WHO Ethical Review Committee and local ethics research review boards approved ethical and obtained written informed consent on Global Ageing and Adult Health (SAGE). All experiments were performed in accordance with relevant guidelines and regulations. All experimental protocols were approved by the Institutional Review Board at China, Three Gorges University. This study was deemed exempt for review by the Institutional Review Board at China, Three Gorges University.

\section{Consent to publication}

Not Applicable.

\section{Competing interests:}

The authors declare that they have no confict of interest.

\section{Funding:}

No funding.

\section{Authors' contributions:}

Z.H. F.Z. and X.Y. wrote the main manuscript text. A.Y.and Y.T. prepared figures 1-3. All authors reviewed the manuscript.

\section{Acknowledgements:}

We thank the CHARLS research and field team and every respondent in the study for their contributions. This work was supported by the Behavioral and Social Research division of the National Institute on Aging of the National Institute of Health (grants 1-R21-AG031372-01, 1-R01-AG037031-01, and 3-R01AG037031-03S1); the Natural Science Foundation of China (grants 70773002, 70910107022, and 71130002), the World Bank (contracts 7145915 and 7159234), and Peking University

\section{References}


1. Global Initiative for Asthma. Global Strategy for Asthma Management and Prevention, 2018. http://www.ginasthma.org (accessed June 10, 2019).

2. McDonald VM, Clark VL, Cordova-Rivera L, Wark PAB, Baines KJ, Gibson PG. Targeting treatable traits in severe asthma: a randomised controlled trial. Eur Respir J. 2020;55(3):1901509..

3. Freitas PD, Xavier RF, McDonald VM, et al. Identification of asthma phenotypes based on extrapulmonary treatable traits. Eur Respir J. 2021;57(1):2000240.

4. Hiles SA, Gibson PG, Agusti A, McDonald VM. Treatable Traits That Predict Health Status and Treatment Response in Airway Disease. J Allergy Clin Immunol Pract. 2021;9(3):1255-1264.e2.

5. Wu WW, Zhang X, Li M, et al. Treatable Traits in Elderly Asthmatics from the Australasian Severe Asthma Network: A Prospective Cohort Study. J Allergy Clin Immunol Pract. 2021;9(7):2770-2782.

6. Chen LK, Woo J, Assantachai P, et al. Asian Working Group for Sarcopenia: 2019 Consensus Update on Sarcopenia Diagnosis and Treatment. J Am Med Dir Assoc. 2020;21(3):300-307.e2.

7. Nelke C, Dziewas R, Minnerup J, Meuth SG, Ruck T. Skeletal muscle as potential central link between sarcopenia and immune senescence. EBioMedicine. 2019;49:381-388.

8. Ohara DG, Pegorari MS, Oliveira Dos Santos NL, de Fatima Ribeiro Silva C, Monteiro RL, Matos AP, et al. Respiratory Muscle Strength as a Discriminator of Sarcopenia in Community-Dwelling Elderly: A Cross-Sectional Study. J Nutr Health Aging. 2018;22:952-958.

9. Landi F, Salini S, Zazzara MB, et al. Relationship between pulmonary function and physical performance among community-living people: results from Look-up 7+ study. J Cachexia Sarcopenia Muscle. 2020;11(7):38-45.

10. Cruz-Jentoft AJ, Bahat G, Bauer J, et al. Sarcopenia: revised European consensus on definition and diagnosis. Age Ageing 2019; 48:16-31.

11. Zhao Y, Hu Y, Smith JP, Strauss J, Yang G. Cohort profile: the China Health and Retirement Longitudinal Study (CHARLS). Int J Epidemiol. 2014;43(1):61-68.

12. Kowal P, Chatterji S, Naidoo N, et al. Data resource profile: the World Health Organization Study on global AGEing and adult health (SAGE). Int J Epidemiol. 2012;41(6):1639-1649.

13. Wen X, Wang M, Jiang CM, Zhang YM. Anthropometric equation for estimation of appendicular skeletal muscle mass in Chinese adults. Asia Pac J Clin Nutr. 2011;20(4):551-556.

14. Delmonico MJ, Harris TB, Lee JS, Visser M, Nevitt M, Kritchevsky SB, et al. Alternative definitions of sarcopenia, lower extremity performance, and functional impairment with aging in older men and women. J Am Geriatr Soc. 2007; 55:769-774

15. Yang $\mathrm{M}, \mathrm{Hu} \mathrm{X}$, Wang $\mathrm{H}$, et al. Sarcopenia predicts readmission and mortality in elderly patients in acute care wards: A prospective study. J Cachexia Sarcopenia Muscle 2017;8:251e-258

16. Hu X, Zhang L, Wang $\mathrm{H}$, et al. Malnutrition-sarcopenia syndrome predicts mortality in hospitalized older patients. Sci Rep 2017;7:3171.

17. Cheng HG, Chen S, McBride O, Phillips MR. Prospective relationship of depressive symptoms, drinking, and tobacco smoking among middle-aged and elderly community-dwelling adults: Results 
from the China Health and Retirement Longitudinal Study (CHARLS). J Affect Disord. 2016;195:136143.

18. Chen H, Xiong P, Chen L, Hao G. Childhood neighborhood quality, friendship, and risk of depressive symptoms in adults: The China health and retirement longitudinal study. J Affect Disord. 2020;276: 732-737.

19. Hu Z, Song X, Hu K, Ruan Y, Zeng F. Association between sleep duration and asthma in different weight statuses (CHNS 2009-2015). Sleep Breath. 2021;25(1):493-502.

20. Hu Z, Li S, Yang A, et al. Delayed hospital admission and high-dose corticosteroids potentially prolong SARS-COV-2 RNA detection duration of patients with COVID-19. Eur J Clin Microbiol Infect Dis. 2021;40(4):841-848.

21. Bone AE, Hepgul N, Kon S, Maddocks M. Sarcopenia and frailty in chronic respiratory disease. Chron Respir Dis. 2017;14(1):85-99.

22. Jones SE, Maddocks M, Kon SS, et al. Sarcopenia in COPD: prevalence, clinical correlates and response to pulmonary rehabilitation. Thorax. 2015;70(3):213-218.

23. Cruz-Jentoft AJ, Sayer AA. Sarcopenia. Lancet. 2019;393(10191):2636-2646.

24. Papadopoulou SK. Sarcopenia: A Contemporary Health Problem among Older Adult Populations. Nutrients. 2020;12(5): 1293.

25. Brown DM, Goljanek-Whysall K. microRNAs: Modulators of the underlying pathophysiology of sarcopenia?. Ageing Res Rev. 2015;24(Pt B):263-273.

26. Yanai K, Kaneko S, Ishii H, et al. MicroRNAs in Sarcopenia: A Systematic Review. Front Med (Lausanne). 2020;7:180.

27. Pascual-Fernández J, Fernández-Montero A, Córdova-Martínez A, Pastor D, Martínez-Rodríguez A, Roche E. Sarcopenia: Molecular Pathways and Potential Targets for Intervention. Int J Mol Sci. 2020;21(22):8844.

28. Specjalski K, Jassem E. MicroRNAs: Potential Biomarkers and Targets of Therapy in Allergic Diseases?. Arch Immunol Ther Exp (Warsz). 2019;67(4):213-223.

29. Zhang L, Zhang X, Zheng J, et al. Depressive symptom-associated IL-1 $\beta$ and TNF-a release correlates with impaired bronchodilator response and neutrophilic airway inflammation in asthma. Clin Exp Allergy. 2019;49(6):770-780.

30. Qaisar R, Karim A, Muhammad T, Shah I. Circulating Biomarkers of Accelerated Sarcopenia in Respiratory Diseases. Biology (Basel). 2020;9(10):322.

31. Chiba Y, Tanabe M, Goto K, Sakai H, Misawa M. Down-regulation of miR-133a contributes to upregulation of Rhoa in bronchial smooth muscle cells. Am J Respir Crit Care Med. 2009;180(8):713719 .

32. Fan J, Kou X, Yang Y, Chen N. MicroRNA-Regulated Proinflammatory Cytokines in Sarcopenia. Mediators Inflamm. 2016;2016:1438686. 
33. Szlejf C, Suemoto CK, Brunoni AR, et al. Depression is Associated With Sarcopenia Due to Low Muscle Strength: Results From the ELSA-Brasil Study. J Am Med Dir Assoc. 2019;20(12):1641-1646.

34. Kirk B, Zanker J, Bani Hassan E, Bird S, Brennan-Olsen S, Duque G. Sarcopenia Definitions and Outcomes Consortium (SDOC) Criteria are Strongly Associated With Malnutrition, Depression, Falls, and Fractures in High-Risk Older Persons. J Am Med Dir Assoc. 2021;22(4):741-745.

35. Alexandre Tda S, Duarte YA, Santos JL, Wong R, Lebrao ML. Sarcopenia according to the European Working Group on Sarcopenia in Older People (EWGSOP) versus dynapenia as a risk factor for mortality in the elderly. J Nutr Health Aging. 2014; 18:751-756.

\section{Figures}

A

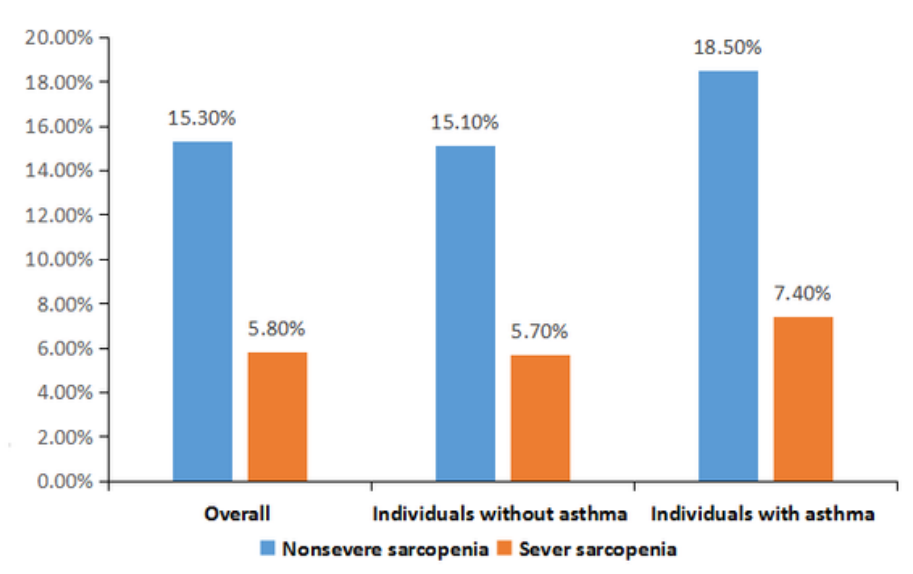

B

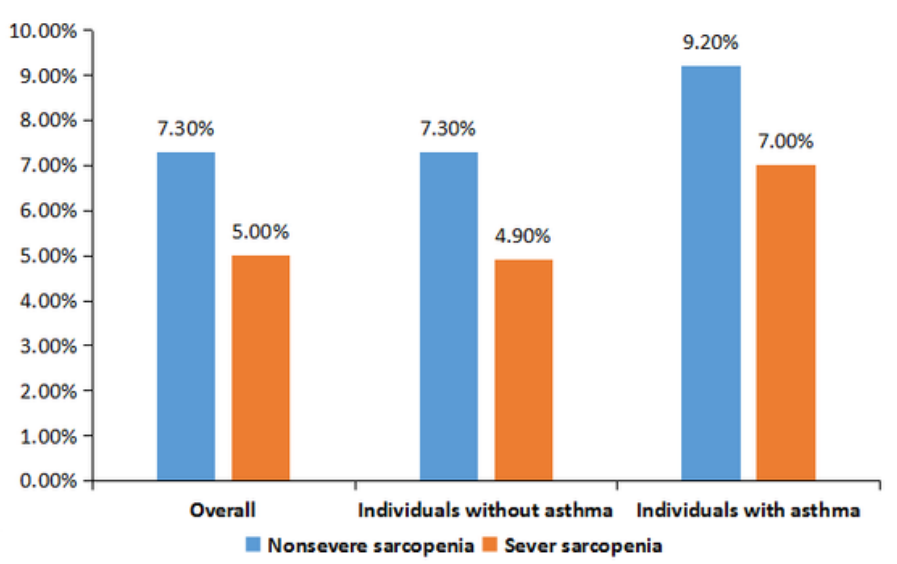

Figure 1

The prevalence of sarcopenia. A. In the China Health and Retirement Longitudinal Study; B. the Study on global AGEing and adult health from China. 


\section{Adjusted mean \& $95 \% \mathrm{Cl}$}

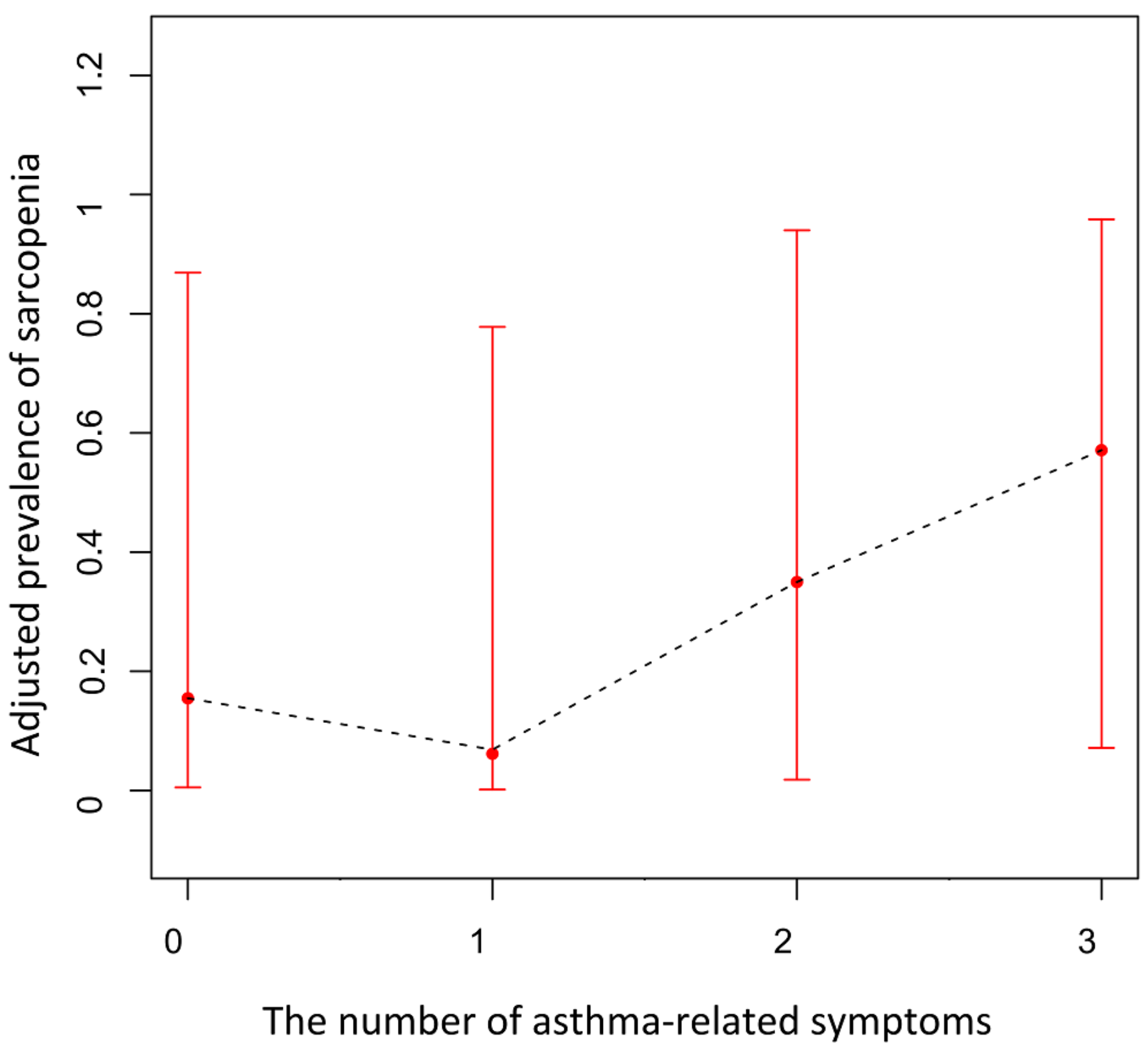

Figure 2

The associations between the prevalence of sarcopenia with the number of asthma-related symptoms in asthmatics from the Study on global AGEing and adult health from China. 
A

Adjusted mean \& $95 \% \mathrm{Cl}$

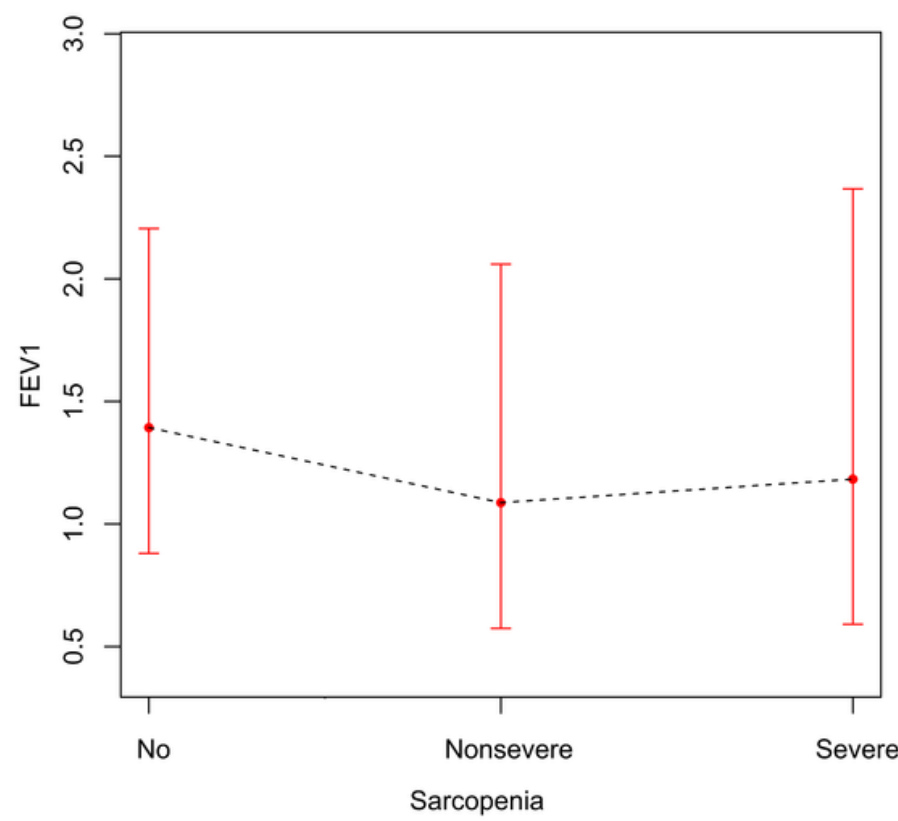

C

Adjusted mean \& $95 \% \mathrm{Cl}$
B

Adjusted mean \& $95 \% \mathrm{Cl}$

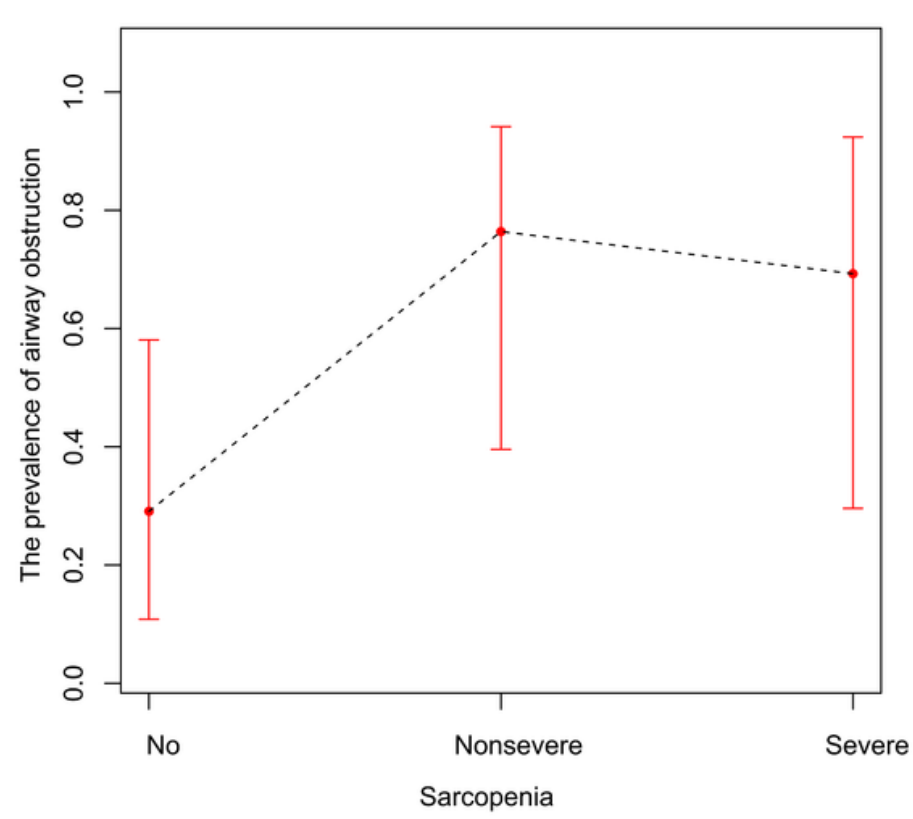

Adiusted mean \& $95 \% \mathrm{Cl}$

Figure 3

The associations between different grade of sarcopenia with lung function in asthmatics from the Study on global AGEing and adult health from China. A: FEV1; B: FEV1/FVC < 0.7; C: PEF; D: FEF 25\%-75\%.

\section{Supplementary Files}

This is a list of supplementary files associated with this preprint. Click to download.

- S1Figure.tif 
- supplyment11.docx

Page 25/25 